\title{
MicroRNA-186 suppresses cell proliferation and metastasis through targeting MAP3K2 in non-small cell lung cancer
}

\author{
TONGHAI HUANG ${ }^{1,4}$, KELIN SHE ${ }^{1}$, GUILIN PENG ${ }^{2,3}$, WEI WANG ${ }^{2,3}$ \\ $\mathrm{JUN} \mathrm{HUANG}^{2,3}$, JINGPEI LI ${ }^{2,3}$, ZHENG WANG ${ }^{4}$ and JIANXING HE ZI-3 $^{1-3}$ \\ ${ }^{1}$ Nanfang Hospital, Southern Medical University, Guangzhou, Guangdong 510515; \\ ${ }^{2}$ State Key Laboratory of Respiratory Disease, National Clinical Research Center for Respiratory Disease; \\ ${ }^{3}$ Department of Thoracic Surgery, The First Affiliated Hospital of Guangzhou Medical University, Guangzhou, \\ Guangdong 510120; ${ }^{4}$ Department of Thoracic Surgery, Shenzhen People's Hospital, Shenzhen, Guangdong 518020, P.R. China
}

Received April 19, 2016; Accepted June 22, 2016

DOI: $10.3892 /$ ijo.2016.3637

\begin{abstract}
MicroRNAs are a class of small endogenous noncoding RNAs that play crucial roles in the initiation and progression of human cancers. miR-186 was found decreased in various human malignancies and function as a tumor suppressor. However, the regulating mechanism of miR-186 in growth and metastasis of human non-small cell lung cancer (NSCLC) is still poorly understood. We investigated the role of miR-186 in the growth and metastasis of human NSCLC. In the present study, we found that miR-186 was significantly decreased in lung cancer tissues and cells. Furthermore, overexpression of miR-186 suppressed lung cancer cell proliferation, migration and invasion, and induced cell apoptosis. Moreover, we found that confirmed mitogen-activated protein kinase kinase kinase 2 (MAP3K2) protein was increased in lung cancer tissues and confirmed that MAP3K2 is a target gene of miR-186. In addition, knockdown of MAP3K2 by RNA interference inhibited lung cancer cell proliferation, migration and invasion, and promoted cell apoptosis in vitro. Furthermore, we observed that the overexpression of MAP3K 2 partially reversed the inhibitory effect of miR-186 on the proliferation and metastasis of A549 and HCC827 cell lines. Taken together, our data indicated that miR-186 regulates lung cancer growth and metastasis through suppressing MAP3K2 expression, at least partly. Therefore, miR-186-MAP3K2 may represent a new and useful potential clinical treatment and diagnosis target for NSCLC.
\end{abstract}

Correspondence to: Professor Jianxing He, Department of Thoracic Surgery, The First Affiliated Hospital of Guangzhou Medical University, no. 151, Yanjiang Road, Guangzhou, Guangdong 510120, P.R. China

E-mail: hejx@vip.163.com

Key words: non-small cell lung cancer, miRNA-186, growth, metastasis, apoptosis, MAP3K2

\section{Introduction}

Lung carcinoma is one of the most common cancers in both male and female and the leading cause of cancer related death all around the world (1). In 2015, there were an estimated more than 222,0000 new cases of lung cancer accounting for $14 \%$ of all new diagnoses from cancer, and leading to an estimated 158,040 cancer deaths, in the United States (2). Moreover, it is anticipated that the incidence of lung cancer will keep increasing in Asian and many developing countries with the high and increasing number of smoking. Rates of lung cancer are mainly determined by smoking patterns, but medical, occupational, and environmental radiation exposures, have also been shown to increase risks of lung cancer (3).

Lung cancer is a heterogeneous disease that has historically been divided into two main types based on the disease patterns and treatment strategies: small cell lung cancer (SCLC) and non-small cell lung cancer (NSCLC) (4,5), and NSCLC comprises approximately $80 \%$ of all lung cancers (6). Although the treatments of lung cancer have advanced and improved greatly, the 5-year survival rate of lung cancer patients is little improved (1). Over the last decades, the local control for early-stage non-small cell lung cancer (NSCLC) has dramatically improved for lung cancer patients $(7,8)$. However, nearly $20 \%$ of early-stage lung cancer patients are developing distant metastasis $(9,10)$. In recent decades, tremendous efforts were made to understand the occurrence and development of lung cancer, but the metastasis mechanisms of NSCLC have not been clarified.

MicroRNAs (miRNAs) are a class of small endogenous non-coding RNAs with approximately 18-22 nucleotides in length. miRNAs can directly bind to the 3 ' untranslated regions (3'-UTRs) of target genes resulting in negatively regulating mRNA stability or suppressing translation $(11,12)$. Increasing number of research has proven that miRNAs are involved in various cellular and molecular processes including growth, apoptosis, differentiation, metabolism and immunity $(13,14)$. In addition, many studies have confirmed that miRNAs play crucial roles in the initiation and progression of human cancers, such as ovarian cancer (15), hepatocellular carcinoma (16), breast cancer (17) and lung carcinoma (18). miR-186 was 
Table I. Primer sequences used for miRNA and mRNA expression analysis.

Name

Primer sequence $\left(5^{\prime}-3^{\prime}\right)$

miR-186-RT
U6-RT
U6-F
U6-R
miR-186-F
Universal-R
MAP3K2-F
MAP3K2-R
GAPDH-F
GAPDH-R

CTCAACTGGTGTCGTGGAGTCGGCAATTCAGTTGAGTTTGGGCT CGCTTCACGAATTTGCGTGTCAT

CTCGCTTCGGCAGCACA

AACGCTTCACGAATTTGCGT

ACACTCCAGCTGGGCAGCAGCACACT

CTCAACTGGTGTCGTGGA

CTTGCTACATGAGCGAATTGTT

AATCTGACGGGTGTATTTCCTA

TGTTCGTCATGGGTGTGAA

ATGGCATGGACTGTGGTCAT

F, forward primer; R, reverse primer; RT, reverse transcription primer.

found deregulated in various human cancers. The expression of miR-186 was observed significantly increased in pancreatic cancer tissues (19). Nevertheless, recent studies reported the expression of miR-186 was decreased in various human malignancies and function as a tumor suppressor $(20,21)$. A previous study showed that miR-186 was significantly decreased in non-small cell lung cancer $(22,23)$. However, the concrete mechanism of miR-186 in lung cancer metastasis remains elusive.

In the present study, we found that miR-186 was significantly decreased in lung cancer tissues. Overexpression of miR-186 suppressed lung cancer cell proliferation, invasion, migration and induced apoptosis by direct targeting of mitogen-activated protein kinase kinase kinase 2 (MAP3K2). Our results revealed the miR-186-MAP3K2 may represent a new potential target for diagnosis and treatment of lung carcinoma.

\section{Materials and methods}

Clinical specimens. A total of 20 cases of NSCLC specimens (20 tumor tissues and 20 matched adjacent normal tissues) were obtained from the Nanfang Hospital, Southern Medical University from 2014 to 2015 . All patients underwent surgery before receiving chemotherapy and/or radiation therapy. All patients were histologically diagnosed according to the clinicopathological criteria of the UICC. The median age of NSCLC patients was 51.7 years (range, 49-68 years). All of the malignant tissues were from stage II-III tumors. The study was approved by the Ethics Committee of the Nanfang Hospital, Southern Medical University, and all volunteers provided informed consents for the use of their specimens in this study.

Cell lines and culture condition. Primary pulmonary epithelial cells (HPAEpiC) were purchased from ScienCell Research Laboratories (Carlsbad, CA, USA) and cultured according to the supplier's instructions using alveolar epithelial cell medium (ScienCell Research Laboratories; cat. \#3201) with 10\% fetal bovine serum (FBS; Gibco, Carlsbad, CA, USA). Human lung cancer cell lines A549, 95-D, HCC827 and NCI-H1650 were obtained from Shanghai Institute of Cell Biology (Shanghai,
China). Four cancer cell lines were cultured in the RPMI-1640 medium (ATCC, Manassas, VA, USA) with 10\% FBS. The media contained $100 \mathrm{UI} / \mathrm{ml}$ penicillin and $100 \mu \mathrm{g} / \mathrm{ml}$ streptomycin. Cells were cultured and maintained in a humidified incubator at $37^{\circ} \mathrm{C}$ and supplemented with $5 \% \mathrm{CO}_{2}$.

Plasmid construction. The 3'-untranslated region (3'-UTR) of MAP3K2 was amplified from human genomic DNA and cloned downstream of psi-CHECK2 vector (Promega, Madison, WI, USA), and termed MAP3K2-3'UTR-WT. Mutations of miR-186 binding sites (AUUCUUU to CAAUCU) were introduced by QuikChange Site-Directed Mutagenesis kit (Stratagene, La Jolla, CA, USA), and termed MAP3K23'UTR-MUT.

For overexpression plasmid cloning, MAP3K2 cDNA was first reverse-transcribed from the RNA of the A549 cells using M-MLV Reverse Transcriptase (Promega) with oligo(dT) primer. The coding region (1860 bp) was then amplified by PCR from cDNA and inserted into the HindIII/BamHI site of the pcDNA3.1 vector (Promega). The following sequences were used: forward primer 5'-AAGCTTATGGATGATCA GCAAGC-3' and reverse primer 5'-GGATCCCTAGTGATA ATGCACAAACAT-3'. Cloned products were confirmed by DNA sequencing.

Cell transfection. A549 or HCC827 $\left(1 \times 10^{4}\right)$ cells/well were seeded in 96-well culture plates. After culture for $24 \mathrm{~h}$, miR-186 mimics (100 mM) (Shanghai GenePharma Co., Ltd., Shanghai, China) or MAP3K2-siRNA (100 mM) (GenePharma) or pcDNA3.1-MAP3K2 $(20 \mu \mathrm{g})$ or equal amount of negative control sequences and vectors were transfected into A549 or HCC827 cells using Lipofectamine 2000 (Beyotime Institute of Biotechnology, Shanghai, China). After cultured for different times, cells were harvested and used in further study.

Quantitative real-time polymerase chain reaction ( $q R T-P C R$ ). Total RNA from tissues or $1 \times 10^{7}$ cells was extracted using TRIzol reagent (Invitrogen Inc., Waltham, MA, USA). SYBR PrimeScript miRNA RT PCR kit (Takara Bio, Dalian China) was used to detect the miR-186 expression level and results were 
normalized to U6. For qRT-PCR, $2 \mu \mathrm{g}$ of total RNA was used for reverse-transcription PCR using M-MLV Reverse Transcriptase (Promega). SYBR-Green Real-Time Master Mix (Toyobo Co., Ltd., Osaka, Japan) was used to analyze the relative expression of MAP3K2 and GAPDH was used as an internal control. All primers were synthesized by Sangon (Sangon Biotech Co., Ltd., Shanghai, China) (Table I). Applied Biosystems 7500 Sequence Detection system (Applied Biosystems, Foster City, CA, USA) was used to perform qRT-PCR and data collection. miR-186 and MAP3K2 expression levels were calculated and normalized using the $2^{-\Delta \Delta \mathrm{Ct}}$ method.

Protein extraction and western blotting. Lung cancer tissues and cells were washed with PBS (pH 7.4) twice and lysed in RIPA lysis and extraction buffer (Thermo Fisher Scientific, Waltham, MA, USA) with $1 \%(\mathrm{v} / \mathrm{v})$ aprotinin (Millipore, Billerica MA, USA) and $1 \%$ (v/v) pepstatin (Millipore) on ice for $20 \mathrm{~min}$. The lysates or homogenates were centrifuged at $12000 \mathrm{x} \mathrm{g}, 4^{\circ} \mathrm{C}$ for $15 \mathrm{~min}$. Then the collected supernatant and protein concentration was determined using a Pierce BCA protein assay kit (Thermo Fisher Scientific). Protein samples were separated by $12 \%$ SDS-PAGE and detected by western blot analysis using rabbit monoclonal anti-MAP3K2 (ab33918; Abcam, Cambridge, MA, USA) and rabbit monoclonal anti-GAPDH (ab181602; Abcam). Goat anti-rabbit IgG H\&L (ab6721; Abcam) secondary antibody with HRP conjugated and results were developed using the ECL technique (SuperSignal West Femto; Pierce, Rockford, IL, USA).

Cell viability assay. The Cell Counting Kit-8 was used to estimate cell proliferation (24). In brief, $1 \times 10^{4}$ A549 or HCC827 cells/well were seeded in 96-well culture plates. After transfected with miR-186 mimics (100 mM) (GenePharma) or MAP3K2-siRNA (100 mM) (GenePharma) or pcDNA3.1MAP3K2 $(20 \mu \mathrm{g})$ for 24,48 and $72 \mathrm{~h}$, cells were treated with CCK-8 solution for $1 \mathrm{~h}$ at room temperature. The absorbance was measured at $450 \mathrm{~nm}$ using a microplate reader Thermo Plate (Rayto Life and Analytical Science Co., Ltd., Shenzhen, China).

Apoptosis assay. The apoptosis of A549 or HCC827 cells transfected with miR-186 mimics or MAP3K2siRNA or pcDNA3.1-MAP3K2 $(20 \mu \mathrm{g})$ was tested using Annexin V-FITC/PI apoptosis detection kit (BD Biosciences, San Jose, CA, USA) by flow cytometry as previously described (24). All experiments were performed in triplicate.

Invasion and migration assay. The invasion and migration ability of A549 or HCC827 cells transfected with miR-186 mimics or MAP3K2-siRNA or pcDNA3.1-MAP3K2 $(20 \mu \mathrm{g})$ was analyzed in 24-well Transwell chambers ( $8 \mu \mathrm{m}$; Millipore) as previously described (25). The invading cells were fixed with $4 \%$ paraformaldehyde in PBS, stained with $0.5 \%$ crystal violet (Sigma-Aldrich, St. Louis, MO, USA) for $15 \mathrm{~min}$ and counted. Invaded or migrated cells were counted under a microscope (IX71; Olympus, Tokyo, Japan) at x200 magnification.

Statistical analysis. All experiments were performed in triplicate and data were expressed as the mean \pm SD. The difference among groups was determined by one-way analysis
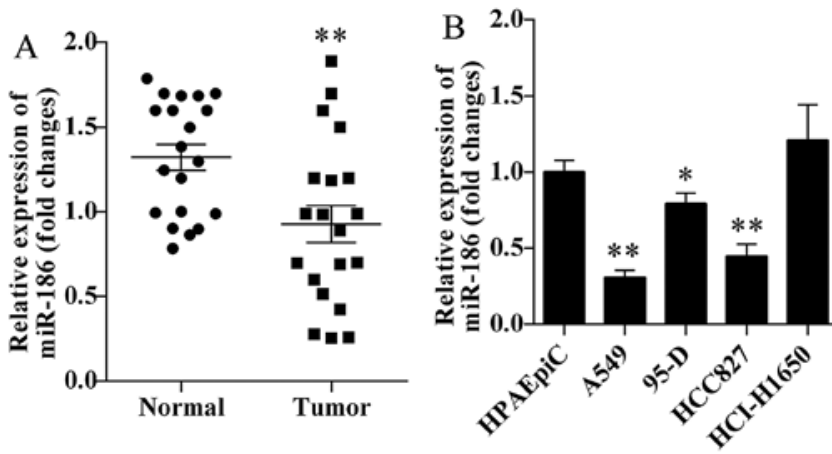

Figure 1. Expression of miR-186 in lung cancer tissues and cell lines. (A) Expression of miR-186 in lung cancer tissues and non-cancerous tissues was examined by qRT-PCR. (B) Expression of miR-186 in lung cancer cells and primary pulmonary epithelial cells was examined by qRT-PCR. ${ }^{* *} \mathrm{P}<0.01$.

of variance (ANOVA) analysis and comparison between two groups was analyzed by a paired/unpaired Student's t-test using SPSS 18.0 program (SPSS Inc., Chicago, IL, USA). The differences were considered statistically significant at $\mathrm{P}<0.05$.

\section{Results}

miR-186 is decreased in human lung cancer tissues and cells. We first investigated the miR-186 expression levels in human lung cancer tissues and corresponding normal tissues. We found that miR-186 was markedly decreased in human lung cancer tissues $(\mathrm{P}=0.0066$; Fig. 1A). Furthermore, we also detected the miR-186 expression levels in four lung cancer cell lines. We found that miR-186 was significantly decreased in A549, 95-D and HCC827 cells (Fig. 1B). Based on the above results, lung cancer cell lines A549 and HCC827 were selected for the present study.

Forced expression of miR-186 induces cell apoptosis and suppresses proliferation. To evaluate the biological functions of miR-186 on cell apoptosis and proliferation, miR-186 mimics were first transfected into A549 and HCC827 cells. Expression of miR-186 was assessed using qRT-PCR analysis and a respective 26.73- and 22.92-fold increased in miR-186 mimic-transfected cells compared with negative control (Fig. 2A). Furthermore, we investigated miR-186 on cell apoptosis using flow cytometric analysis. We found that more apoptotic cells were observed in A549 (Fig. 2B) and HCC827 (Fig. 2C) cells after forced expression of miR-186. The apoptosis rates of A549 cells transfected with miR-186 mimics and $\mathrm{NC}$ were $14.2 \pm 1.2$ and $5.7 \pm 0.9 \%$, respectively (Fig. 2D). In addition, the apoptosis rates of $\mathrm{HCC} 827$ cells transfected with miR-186 mimics and NC were 15.6 \pm 1.1 and 9.6 $\pm 1.3 \%$, respectively (Fig. 2D). Finally, we studied the role of miR-186 on proliferation using CCK-8 assay. We found that overexpression of miR-186 exhibited significantly lower proliferation rates of A549 (Fig. 2E) and HCC827 (Fig. 2F) cells.

Overexpression of miR-186 inhibits cell migration and invasion. Migration and invasion abilities of A549 and HCC827 cells after transfected with miR-186 mimics were assessed using Transwell assay. Transfection of miR-186 mimics markedly reduced the number of A549 (Fig. 3A and B) and HCC827 

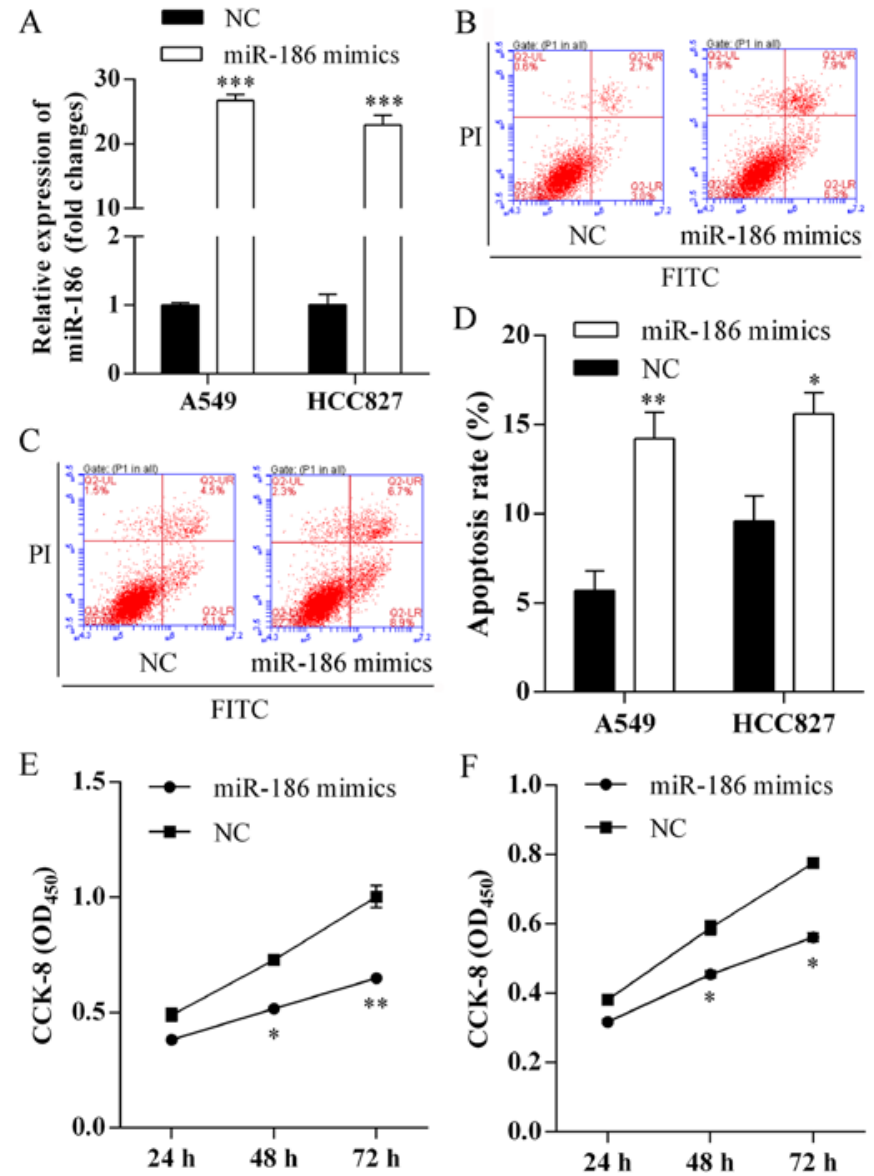

Figure 2. miR-186 inhibits growth and promotes apoptosis of lung cancer cells in vitro. (A) miR-186 expression level was tested by qRT-PCR in A549 and HCC827 cells transfected with miR-186 mimics and negative control. (B) Flow cytometric analysis of A549 cell apoptosis after miR-186 transfection. (C) Flow cytometric analysis of HCC827 cell apoptosis after miR-186 transfection. (D) The apoptosis rates of A549 and HCC827 cells after miR-186 transfection. (E) The effect of miR-186 on A549 cell proliferation was determined by CCK- 8 assay at 24,48 and $72 \mathrm{~h}$. (F) The effect of miR-186 on HCC 827 cell proliferation was determined by CCK- 8 assay at 24,48 and 72 h. ${ }^{* * *} \mathrm{P}<0.001$.

(Fig. 3C and D) cells that passed through the Transwell chamber. The results indicated that miR-186 might inhibit cell migration and invasion.

miR-186 directly targets MAP3K2 3'UTR. To evaluate the molecular mechanism by which miR-186 inhibits the proliferation and metastasis of A549 and HCC 827 cells, we first detected the protein expression level of MAP3K2 in human lung cancer tissues and compared non-cancerous tissues using western blot analysis. We found that MAP3K2 was increased in tumor tissues (Fig. 4A). Furthermore, overexpression of miR-186 suppressed the protein expression of MAP3K2 in A549 and HCC827 cells (Fig. 4B). Moreover, we predicted that the MAP3K2 is one of the target genes of miR-186 in human using the predication tool TargetScan and miRanda. miR-186 has one predictive target site in the MAP3K2 3' UTR (Fig. 4C). In addition, the luciferase activity of MAP3K2 3'UTR-WT was significantly decreased upon overexpressed by miR-186 in A549 cells, whereas, its mutant type was not changed (Fig. 4D). Taken together, our data indicated that MAP3K2 is a direct target gene of miR-186.
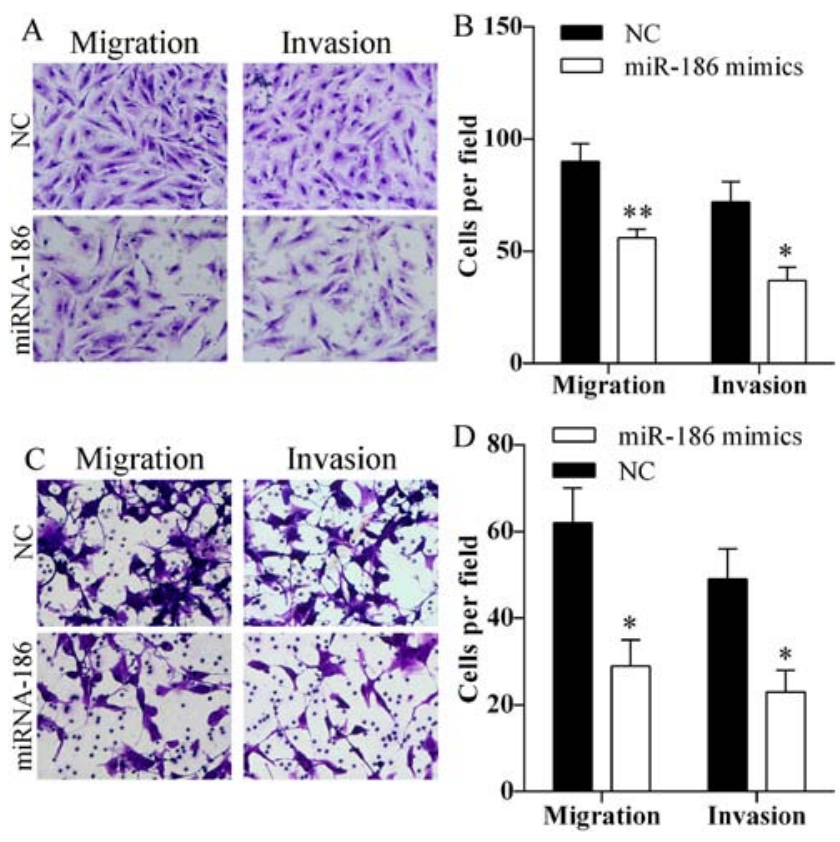

Figure 3. miR-186 inhibits migration and invasion of lung cancer cells in vitro. (A) The effect of miR-186 on A549 cell migration and invasion was determined by Transwell assay. (B) The average invaded or migrated A549 cells in different experimental groups. (C) The effect of miR-186 on HCC827 cell migration and invasion was determined by Transwell assay. (D) The average invaded or migrated HCC827 cells in different experimental groups. ${ }^{* *} \mathrm{P}<0.01$.

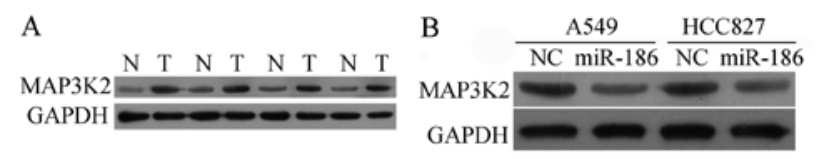

C 3' UCGGGUUUUCCUCUUAAGAAAC 5' miR-186 508: 5' CUUCUUAUAUCUGUAUUUCUUUUA 3' MAP3K2 WT 508: 5' CUUCUUAUAUCUGUCAAUCUAA 3' MAP3K2 MUT

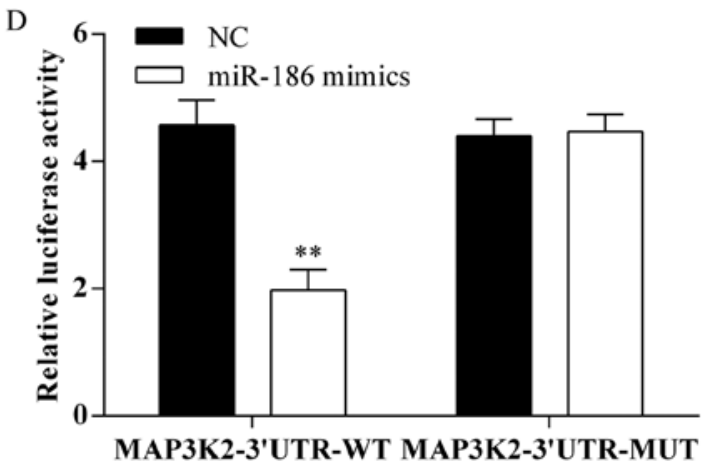

Figure 4. miR-186 directly targets MAP3K2. (A) Western blot analysis of the MAP3K2 protein expression in lung cancer tissues and non-cancerous tissues. (B) Western blot analysis of the MAP3K2 protein expression level in A549 and HCC827 cells after miR-186 transfection. (C) Sequence alignment between miR-186 and the 3'UTR of human MAP3K2. (D) The effect of miR-186 on the activity of luciferase reporter containing either MAP3K23'UTR-WT or MAP3K2-3'UTR-MUT was tested by luciferase reporter gene assays. ${ }^{* *} \mathrm{P}<0.01$.

Silence MAP3K2 expression induces cell apoptosis and suppresses proliferation. To evaluate the role of MAP3K2 in miR-186-induced cell apoptosis and proliferation, MAP3K2- 

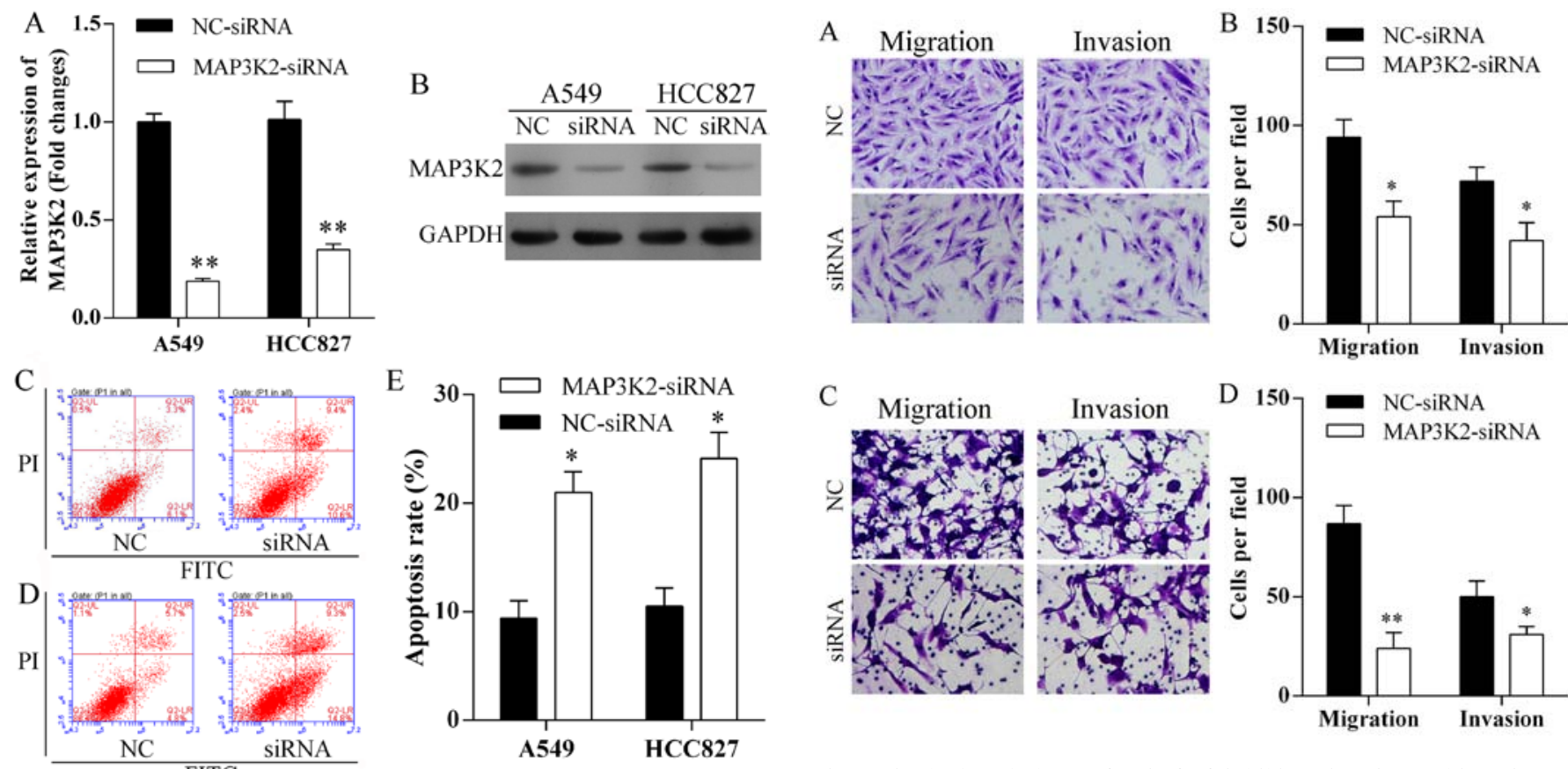

Figure 6. The knock-down of MAP3K2 inhibits migration and invasion of lung cancer cells in vitro. (A) The effect of MAP3K2 on A549 cell migration and invasion was determined by Transwell assay. (B) The average invaded or migrated A549 cells in different experimental groups. (C) The effect of MAP3K2 on HCC827 cell migration and invasion was determined by Transwell assay. (D) The average invaded or migrated HCC827 cells in different experimental groups. ${ }^{* *} \mathrm{P}<0.01$
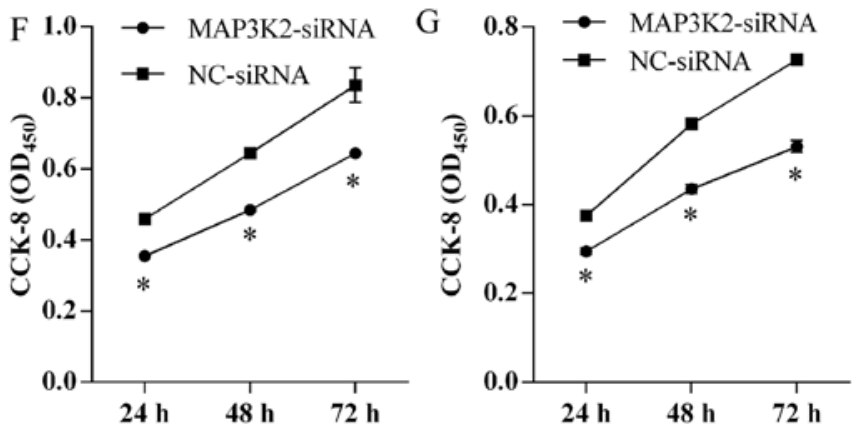

Figure 5. The knock-down of MAP3K2 inhibits growth and promotes apoptosis of lung cancer cells in vitro. (A) MAP3K 2 mRNA expression level was tested by qRT-PCR in A549 and HCC827 cells transfected with MAP3K2siRNA. (B) MAP3K2 protein expression level was tested by western blot analysis in A549 and HCC827 cells transfected with MAP3K2-siRNA. (C) Flow cytometric analysis of A549 cell apoptosis after MAP3K2-siRNA transfection. (D) Flow cytometric analysis of HCC827 cell apoptosis after MAP3K2-siRNA transfection. (E) The apoptosis rates of A549 and HCC827 cells after MAP3K2-siRNA transfection. (F) A549 cell proliferation was determined by CCK- 8 assay at 24, 48 and $72 \mathrm{~h}$ after MAP3K2-siRNA transfection. (G) HCC827 cell proliferation was determined by CCK-8 assay at 24 , 48 and $72 \mathrm{~h}$ after MAP3K2-siRNA transfection. ${ }^{* *} \mathrm{P}<0.01$.

siRNA was transfected into A549 and HCC827 cells to specifically knock down MAP3K2 expression. MAP3K2 expression level was remarkably repressed at mRNA (Fig. 5A) and protein (Fig. 5B) level in A549 and HCC827 cells after MAP3K2-siRNA transfection. Furthermore, increased number of apoptotic cells were observed in A549 (Fig. 5C) and HCC827 (Fig. 5D) cells following repressed MAP3K2 expression. The apoptosis rates of A549 cells transfected with MAP3K2-siRNA and NC-siRNA were 20.0 \pm 1.4 and $9.4 \pm 1.2 \%$, respectively (Fig. 5E). In addition, the apoptosis rates of $\mathrm{HCC} 827$ cells transfected with MAP3K2-siRNA and NC-siRNA were $24.1 \pm 1.9$ and $10.5 \pm 1.3 \%$, respectively (Fig. 5E). Finally, we found that the knock-down of MAP3K2 exhibited significantly lower proliferation rates of A549 (Fig. 5F) and HCC827 (Fig. 5G) cells.

Silencing MAP3K2 expression inhibits cell migration and invasion. Transfection of MAP3K2-siRNA markedly reduced the number of A549 (Fig. 6A and B) and HCC827 (Fig. 6C and D) cells that passed through the Transwell chamber.

MAP3K2 ameliorated the inhibitory effect of miR-186 on cell proliferation and metastasis. For further study, MAP3K2 was overexpressed after transfection of pcDNA3.1-MAP3K2 vectors in miR-186 A549 and HCC827 cells (Fig. 7A). In addition, overexpression of MAP3K2 in A549 (Fig. 7B) and HCC827 (Fig. 7C) overexpressing miR-186 cells significantly decreased the inhibitory effect of miR-186 on lung cancer cell proliferation (Fig. 7B and C). Moreover, overexpression of MAP3K2 in A549 and HCC827 overexpressing miR-186 cells significantly reduced miR-186 induced cell apoptosis (Fig. 7D and E). Moreover, overexpression of MAP3K2 in A549 (Fig. 7F and G) and HCC827 (Fig. H and I) overexpressing miR-186 cells significantly decreased the inhibitory effect of miR-186 on lung cancer cell migration and invasion (Fig. 7F-I).

\section{Discussion}

In recent years, increased number of studies found that miRNAs played critical roles in fundamental cellular processes $(26,27)$, and an increasingly recognized that miRNAs are abnormally expressed and involved in a variety of human cancers $(16,18,24,28)$. The expression of miR-186 has been found disordered in different human cancers. Zhang et al (19) found that miR-186 was significantly upregulated in pancreatic cancer and might play a critical role in diagnosis 
A

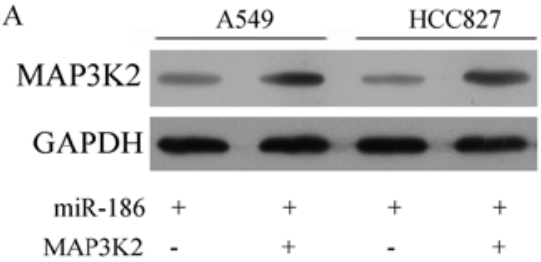

D

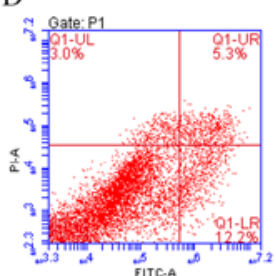

miR-186+Vector

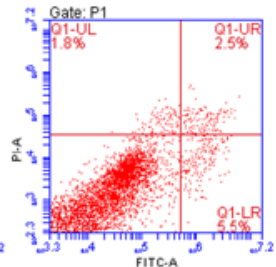

A549

F

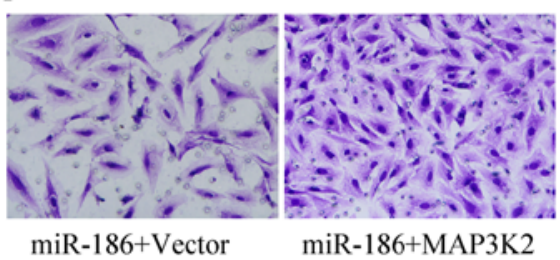

Migration

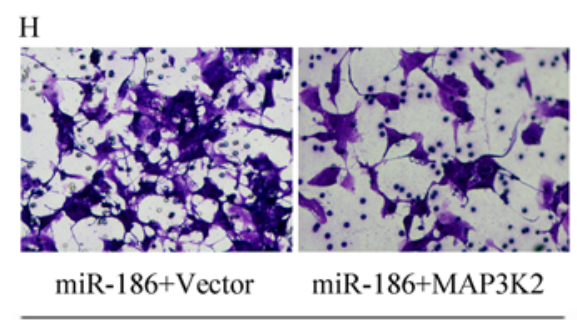

Migration
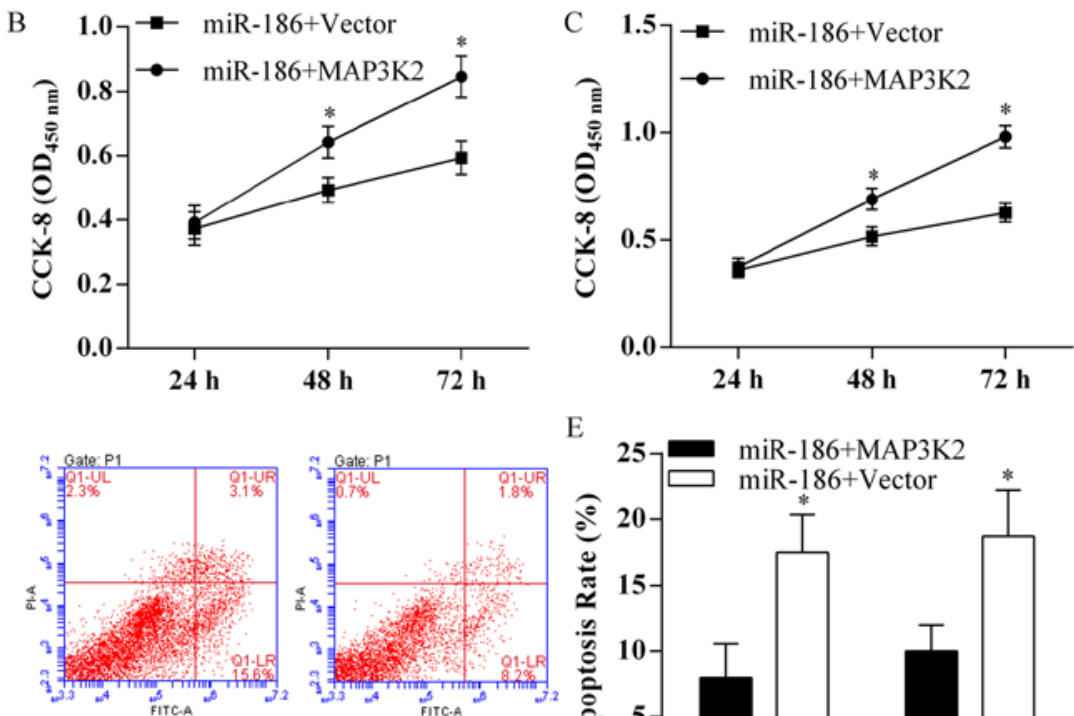

miR-186+Vector

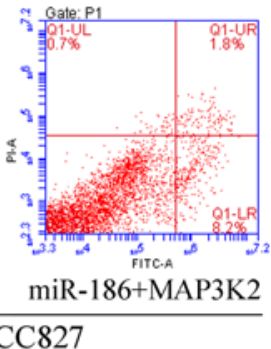

E

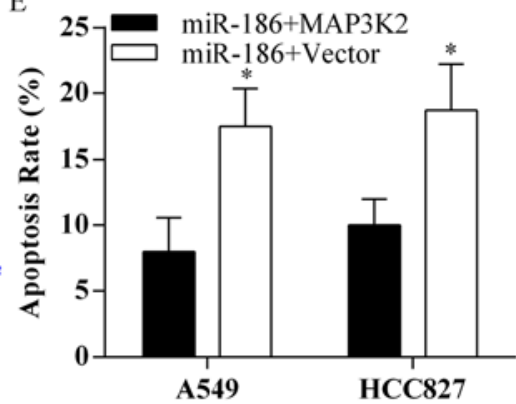

G

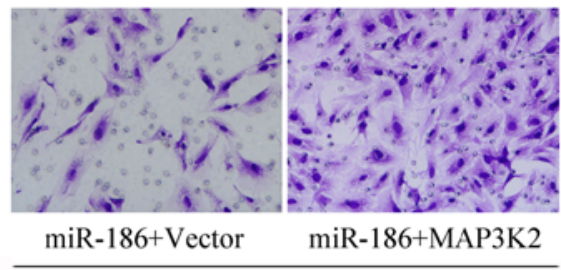

Invasion

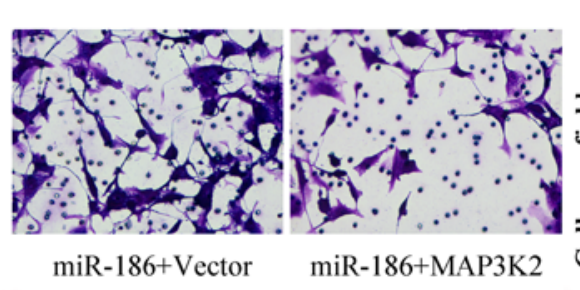

Invasion

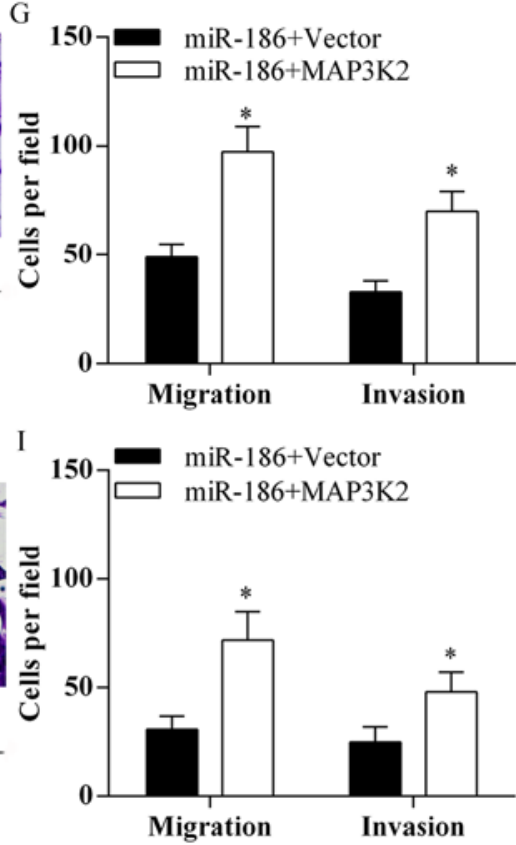

Figure 7. MAP3K2 ameliorated the inhibitory effect of miR-186 on cell proliferation and metastasis. (A) MAP3K2 expression levels in A549 and HCC827 cells were detected using western blot analysis. (B and C) A549 and HCC827 cell proliferation was determined by CCK-8 assay at 24, 48 and $72 \mathrm{~h}$. (D and E) A549 and HCC827 cell apoptosis rates were determined through flow cytometry. (F and G) A549 cell migration and invasion abilities in different experimental groups. (H and I) HCC827 cell migration and invasion abilities in different experimental groups.

and prognosis of pancreatic cancer. Whereas, miR-186 was discovered decreased in lung adenocarcinoma and correlated with patient survival $(22,23)$. Furthermore, tumor-suppressive effects of miR-186 in medulloblastomas (29), endometrial (20) and prostate cancer (21) were also observed. However, little is known of the potential role of miR-186 on growth and metastasis of human non-small cell lung cancer.

In the present study, we first analyzed the expression of miR-186 in 20 lung cancer tissues and four cancer cell lines. Results showed that miR-186 was significantly decreased in cancer tissues and cell lines. The results were similar with previous research $(22,23)$. To understand the biological role of miR-186 in lung cancer, the impacts of miR-186 on cell prolif- eration, metastasis and apoptosis were analyzed using CCK-8 assay, Transwell assay and flow cytometry apoptosis assay, respectively. Here, we found that overexpression of miR-186 induced A549 and HCC827 cell apoptosis and suppressed cell proliferation. Also, upregulation of miR-186 inhibited A549 and HCC827 cell migration and invasion. These findings suggested miR-186 played important roles in occurrence and metastasis of human non-small cell lung cancer and might be a useful diagnostic and predictive biomarker.

Subsequently, to understand the mechanism of miR-186 in regulating cell growth and metastasis, we demonstrated that mitogen-activated protein kinase kinase kinase 2 (MAP3K2) is a direct target of miR-186 in human lung cancer. MAP3K2, 
a member of serine/threonine protein kinase family in MAPK signaling pathway, is able to activate c-Jun N-terminal kinase (JNK) and ERK5 and preferentially activates other kinases involved in the MAP kinase signaling pathway $(30,31)$. MAP3K2 was found to have important roles in epidermal growth factor (EGF), T-cell receptor and fibroblast growth factor 2 (FGF-2) signaling pathways in MAP3K2 knockout mice $(30,32,33)$. A previous study found that MAP3K2 is able to discriminate tumor from normal cells (34) and MAP3K2 contributed to growth of human hepatocellular carcinoma (35), and miR-520b suppressed hepatoma cell proliferation by targeting MEKK2 (MAP3K2) (35), suggesting that MAP3K2 may play important roles in the development of human cancer. In the present study, we found that MAP3K2 protein was significantly increased in human lung cancer tissues and cell lines. Furthermore, luciferase assays and western blot analysis confirmed that miR-186 directly targets MAP3K2 in lung cancer. In addition, knockdown of MAP3K2 by RNA interference assay markedly reduced the expression of MAP3K2 and suppressed lung cancer cell proliferation, migration and invasion and induced cell apoptosis. All the results indicate that decreased expression of miR-186 promotes cell proliferation and suppresses cell apoptosis capacity of human lung cancer through the MAP3K2 -mediated signal pathway.

In conclusion, we demonstrated that miR-186 is decreased in human lung cancer tissues and cell lines, which targets MAP3K2 directly. miR-186 mimics transfection and knockdown of MAP3K2 inhibited cell proliferative and metastasis of A549 and HCC827 cells and promoted cell apoptosis. Based on the above, the present study may represent a novel indicator of poor prognosis in human lung cancer, and miR-186-MAP3K2 may represent a potential therapeutic target for diagnosis and therapy of human lung cancer.

\section{References}

1. Siegel R, Naishadham D and Jemal A: Cancer statistics, 2013. CA Cancer J Clin 63: 11-30, 2013.

2. Smith RA, Manassaram-Baptiste D, Brooks D, Doroshenk M, Fedewa S, Saslow D, Brawley OW and Wender R: Cancer screening in the United States, 2015: A review of current American cancer society guidelines and current issues in cancer screening. CA Cancer J Clin 65: 30-54, 2015.

3. Novaes FT, Cataneo DC, Ruiz Jr RL, Defaveri J, Michelin OC and Cataneo AJ: Lung cancer: histology, staging, treatment and survival. J Bras Pneumol 34: 595-600, 2008.

4. Youlden DR, Cramb SM and Baade PD: The International Epidemiology of lung cancer: Geographical distribution and secular trends. J Thorac Oncol 3: 819-831, 2008.

5. Johnson JL, Pillai S and Chellappan SP: Genetic and biochemical alterations in non-small cell lung cancer. Biochem Res Int 2012: 940405, 2012.

6. Jemal A, Bray F, Center MM, Ferlay J, Ward E and Forman D: Global cancer statistics. CA Cancer J Clin 61: 69-90, 2011.

7. Ginsberg RJ and Rubinstein LV: Randomized trial of lobectomy versus limited resection for T1 N0 non-small cell lung cancer. Lung Cancer Study Group. Ann Thorac Surg 60: 615-622; discussion 622-613, 1995.

8. Timmerman R, Paulus R, Galvin J, Michalski J, Straube W, Bradley J, Fakiris A, Bezjak A, Videtic G, Johnstone D, et al: Stereotactic body radiation therapy for inoperable early stage lung cancer. JAMA 303: 1070-1076, 2010.

9. Chi A, Liao Z, Nguyen NP, Xu J, Stea B and Komaki R: Systemic review of the patterns of failure following stereotactic body radiation therapy in early-stage non-small-cell lung cancer: clinical implications. Radiother Oncol 94: 1-11, 2010.
10. Martini N, Bains MS, Burt ME, Zakowski MF, McCormack P Rusch VW and Ginsberg RJ: Incidence of local recurrence and second primary tumors in resected stage I lung cancer. J Thorac Cardiovasc Surg 109: 120-129, 1995.

11. Bartel DP: MicroRNAs: Genomics, biogenesis, mechanism, and function. Cell 116: 281-297, 2004.

12. Bartel DP and Chen CZ: Micromanagers of gene expression: The potentially widespread influence of metazoan microRNAs. Nat Rev Genet 5: 396-400, 2004.

13. Chen CZ, Li L, Lodish HF and Bartel DP: MicroRNAs modulate hematopoietic lineage differentiation. Science 303: 83-86, 2004.

14. Kim VN: MicroRNA biogenesis: Coordinated cropping and dicing. Nat Rev Mol Cell Biol 6: 376-385, 2005.

15. Zhang S, Lu Z, Unruh AK, Ivan C, Baggerly KA, Calin GA, Li Z, Bast RC Jr and Le XF: Clinically relevant microRNAs in ovarian cancer. Mol Cancer Res 13: 393-401, 2015.

16. Zhao N, Sun H, Sun B, Zhu D, Zhao X, Wang Y, Gu Q, Dong X, Liu F, Zhang Y, et al: miR-27a-3p suppresses tumor metastasis and VM by down-regulating VE-cadherin expression and inhibiting EMT: An essential role for Twist-1 in HCC. Sci Rep 6: 23091, 2016.

17. Gomes BC, Santos B, Rueff J and Rodrigues AS: Methods for studying MicroRNA expression and their targets in formalinfixed, paraffin-embedded (FFPE) breast cancer tissues. Methods Mol Biol 1395: 189-205, 2016.

18. Kim G, An HJ, Lee MJ, Song JY, Jeong JY, Lee JH and Jeong HC: Hsa-miR-1246 and hsa-miR-1290 are associated with stemness and invasiveness of non-small cell lung cancer. Lung Cancer 91: 15-22, 2016.

19. Zhang Y, Li M, Wang H, Fisher WE, Lin PH, Yao Q and Chen C: Profiling of 95 microRNAs in pancreatic cancer cell lines and surgical specimens by real-time PCR analysis. World J Surg 33: 698-709, 2009.

20. Myatt SS, Wang J, Monteiro LJ, Christian M, Ho KK, Fusi L, Dina RE, Brosens JJ, Ghaem-Maghami S and Lam EW: Definition of microRNAs that repress expression of the tumor suppressor gene FOXO1 in endometrial cancer. Cancer Res 70: 367-377, 2010.

21. Erdmann K, Kaulke K, Thomae C, Huebner D, Sergon M, Froehner M, Wirth MP and Fuessel S: Elevated expression of prostate cancer-associated genes is linked to down-regulation of microRNAs. BMC Cancer 14: 82-82, 2014.

22. Cai J, Wu J, Zhang H, Fang L, Huang Y, Yang Y, Zhu X, Li R and Li M: miR-186 downregulation correlates with poor survival in lung adenocarcinoma, where it interferes with cell-cycle regulation. Cancer Res 73: 756-766, 2013.

23. Cui G, Cui M, Li Y, Liang Y, Li W, Guo H and Zhao S: MiR-186 targets ROCK1 to suppress the growth and metastasis of NSCLC cells. Tumour Biol 35: 8933-8937, 2014.

24. Ruan WD, Wang P, Feng S, Xue Y and Zhang B: MicroRNA-497 inhibits cell proliferation, migration, and invasion by targeting AMOT in human osteosarcoma cells. Onco Targets Ther 9: 303-313, 2016.

25. Wang J, Liu G, Li Q, Wang F, Xie F, Zhai R, Guo Y, Chen T, Zhang N, Ni W, et al: Mucin1 promotes the migration and invasion of hepatocellular carcinoma cells via JNK-mediated phosphorylation of Smad2 at the C-terminal and linker regions. Oncotarget 6: 19264-19278, 2015.

26. Tian L, Fang YX, Xue JL and Chen JZ: Four microRNAs promote prostate cell proliferation with regulation of PTEN and its downstream signals in vitro. PLoS One 8: e75885, 2013.

27. Brennecke J, Hipfner DR, Stark A, Russell RB and Cohen SM: bantam encodes a developmentally regulated microRNA that controls cell proliferation and regulates the proapoptotic gene hid in Drosophila. Cell 113: 25-36, 2003.

28. Hauser B, Zhao Y, Pang X, Ling Z, Myers E, Wang P, Califano J and $\mathrm{Gu}$ X: Functions of MiRNA-128 on the regulation of head and neck squamous cell carcinoma growth and apoptosis. PLoS One 10: e0116321, 2015.

29. Lv SQ, Kim YH, Giulio F, Shalaby T, Nobusawa S, Yang H, Zhou Z, Grotzer $M$ and Ohgaki $H$ : Genetic alterations in microRNAs in medulloblastomas. Brain Pathol 22: 230-239, 2012.

30. Su B, Cheng J, Yang J and Guo Z: MEKK2 is required for T-cell receptor signals in JNK activation and interleukin-2 gene expression. J Biol Chem 276: 14784-14790, 2001. 
31. Chayama K, Papst PJ, Garrington TP, Pratt JC, Ishizuka T, Webb S, Ganiatsas S, Zon LI, Sun W, Johnson GL, et al: Role of MEKK2-MEK5 in the regulation of TNF-alpha gene expression and MEKK2-MKK7 in the activation of c-Jun N-terminal kinase in mast cells. Proc Natl Acad Sci USA 98: 4599-4604, 2001.

32. Schaefer BC, Ware MF, Marrack P, Fanger GR, Kappler JW, Johnson GL and Monks CR: Live cell fluorescence imaging of T cell MEKK2: Redistribution and activation in response to antigen stimulation of the $\mathrm{T}$ cell receptor. Immunity 11: 411-421, 1999 .

33. Sun W, Wei X, Kesavan K, Garrington TP, Fan R, Mei J, Anderson SM, Gelfand EW and Johnson GL: MEK kinase 2 and the adaptor protein Lad regulate extracellular signal-regulated kinase 5 activation by epidermal growth factor via Src. Mol Cell Biol 23: 2298-2308, 2003.
34. Cazares LH1, Troyer D, Mendrinos S, Lance RA, Nyalwidhe JO, Beydoun HA, Clements MA, Drake RR and Semmes OJ: Imaging mass spectrometry of a specific fragment of mitogenactivated protein kinase/extracellular signal-regulated kinase kinase kinase 2 discriminates cancer from uninvolved prostate tissue. Clin Cancer Res 15: 5541-5551, 2009.

35. Zhang W, Kong G, Zhang J, Wang T, Ye L and Zhang X: MicroRNA-520b inhibits growth of hepatoma cells by targeting MEKK2 and cyclin D1. PLoS One 7: e31450, 2012. 